\title{
Malformación cavernomatosa: Revisión de una patología clásica
}

\author{
Dres. Adolfo Aliaga $Q^{(1)}$, Tamara Palavecino $B^{(1)}$ Rodrigo Espinoza $A^{(1)}$ Holvis Dellien $Z^{(2)}$
}

1. Residente de Radiología. Hospital Clínico Universidad de Chile.

2. Neurorradiólogo. Centro Imagenología. Hospital Clínico Universidad de Chile.

\section{Cavernomatous malformations: A classical disease review}

\begin{abstract}
Cavernomatous malformations (CM) are mamelonated vascular hamartomas, initially described by Virchow, which are considered the most common vascular malformation after developmental venous anomalies, with a prevalence of $0.5-0.7 \%$ in the overall population, without gender predilection. In imaging they show a broad range of dynamic behavior given their different levels of growth, possible regression, de novo formation and the risk of bleeding. In these circumstances, magnetic res onance imaging (MRI), especially T2 gradient echo sequence (T2GRE) and susceptibility weighted imaging (SWI), become very important for the detection and follow-up/monitoring of CM.

This article reviews and updates the epidemiological and pathological features of CM; as well as presenting its various locations and characteristics, so that the diagnosis is not complicated.

Keywords: Cavernomatous malformations, MRI, Susceptibility sequence.
\end{abstract}

Resumen. Las malformaciones cavernomatosas $(M C)$ son hamartomas vasculares de aspecto mamelonado, inicialmente descritos por Virchow, que son consideradas la malformación vascular más frecuente, después de las anomalías del desarrollo venoso, con una prevalencia de 0,5-0,7\% en la población general, sin predilección entre géneros. Se presentan con un amplio comportamiento dinámico en las imágenes dado su diverso grado de crecimiento, la posible regresión, la formación de novo y el riesgo de sangrado. En estas circunstancias, la resonancia magnética (RM), especialmente las potenciaciones en T2 gradiente (T2GRE) y susceptibilidad (SWI), cobran gran importancia en su búsqueda y seguimiento.

Se revisa y actualiza su epidemiología y patología; además se presentan sus diversas ubicaciones y características, de tal forma que su diagnóstico no sea complejo.

Palabras clave: Malformación cavernomatosa, Resonancia magnética, Secuencia susceptibilidad.

Aliaga A, et al. Malformación cavernomatosa: Revisión de una patología clásica. Rev Chil Radiol 2013; 19(2): 117-124. Correspondencia: Dr. Holvis Dellien / holvisrx@yahoo.es

Trabajo recibido el 08 de agosto de 2013, aceptado para publicación el 30 de septiembre de 2013.

\section{Introducción}

Las malformaciones cavernosas (MC) son hamartomas vasculares de aspecto mamelonado, inicialmente descritos por Rudolf Virchow en 1863, que forman parte de una de las cuatro malformaciones vasculares del sistema nervioso central (SNC), descritas por Russel y Rubinstein ${ }^{(1)}$.

Conocidas también como cavernomas, angiomas cavernosos y otras denominaciones, son lesiones caracterizadas por la presencia de espacios sinusoidales recubiertos de endotelio, conteniendo restos hemáticos en diferentes estadios de degradación, marginadas por una pseudocápsula de tejido gliótico cargado de hemosiderina. Entre estas cavidades típicamente no existe parénquima cerebral ${ }^{(1)}$.
Pueden encontrarse en cualquier estructura que tenga vascularización, siendo supratentoriales la mayor parte de las lesiones alojadas en el SNC (especialmente en lóbulos frontal y temporal).

\section{Epidemiología}

Es considerada la malformación vascular más frecuente después de las anomalías del desarrollo venoso (angiomas venosos) ${ }^{(2)}$. Se describe una prevalencia de 0,5-0,7\% en la población general, estimada a través de estudios necrópsicos y de RM. La enfermedad no tiene predilección por género y el $25 \%$ de los afectados se encuentra en la población infantil(3,4,5). Se presume una mayor detección de $\mathrm{MC}$ en los últimos años relacionada con el acceso más fácil a estudios imagenológicos ${ }^{(6)}$. 
Los casos de MC familiar son menos frecuentes, sin embargo, se ha descrito una mayor proporción de casos familiares en sujetos con ascendencia hispanoamericana, especialmente en mexicanos ${ }^{(7,8)}$.

En el $75 \%$ de los casos se identifica una lesión solitaria, mientras que entre los casos de tipo familiar, el $62 \%$ se presenta con lesiones múltiples.

Topográficamente, la mayor parte de las lesiones son supratentoriales, de ubicación preferente en lóbulos frontales y temporales. También se las puede encontrar, con menor frecuencia, en médula espinal y retina y, según publicaciones de casos esporádicos, en localizaciones inhabituales como el trayecto de la vía óptica y de otros nervios craneales, las hojas durales y espacios intraventriculares ${ }^{(9-12)}$.

\section{Etiopatogenia}

La mayor cantidad de personas afectadas posee la variedad esporádica (80\%), cuya aparición se ha descrito en relación a infecciones virales, trauma y terapia con radiación; un porcentaje menor posee la presentación de múltiples lesiones en una condición familiar.

Se ha planteado que las MC esporádicas, especialmente las asociadas a otra malformación vascular (anomalías del desarrollo venoso), se formarían de novo secundariamente a fenómenos microhemorrágicos resultantes de fluctuaciones de la presión venosa local en lechos vasculares anormalmente más frágiles, que inducirían alteraciones hemodinámicas locales mediadas por numerosos factores angiogénicos promotores de la formación reactiva y coalescencia de nuevos vasos. Alternativamente, cambios hemodinámicos tales como restricciones al drenaje venoso local y apertura de conexiones (shunts) arteriovenosas preexistentes, favorecerían su crecimiento ${ }^{(13)}$.

Ha sido descrita una alteración genética en los casos del tipo familiar, transmitida por herencia autosómica dominante con penetrancia incompleta y expresión variable, de a lo menos 3 loci identificados como CCM 1-3, ubicados en 7q (CCM1), 7p (CCM2) y $3 q$ (CCM3), los que se han estudiado en modelos animales que poseen alguno de estos genes alterados (knockout) $^{(14,15)}$ (Figura 1).

De esta forma, se han identificado anomalías en los procesos de adhesión celular, migración y proliferación endotelial modulados por los factores de crecimiento VEGF, $\beta F G F$ y TGF $\alpha$, entre otros. Dichos modelos de transducción de señales han permitido a varios laboratorios de investigación planificar el desarrollo de terapias enfocadas específicamente a la alteración de gen CCM2 (que codifica la proteína llamada malcavernina), como por ejemplo, el uso de Simvastatina ${ }^{(16)}$.

Macroscópicamente, las MC se muestran como una masa bien definida, lobulada, de coloración azul

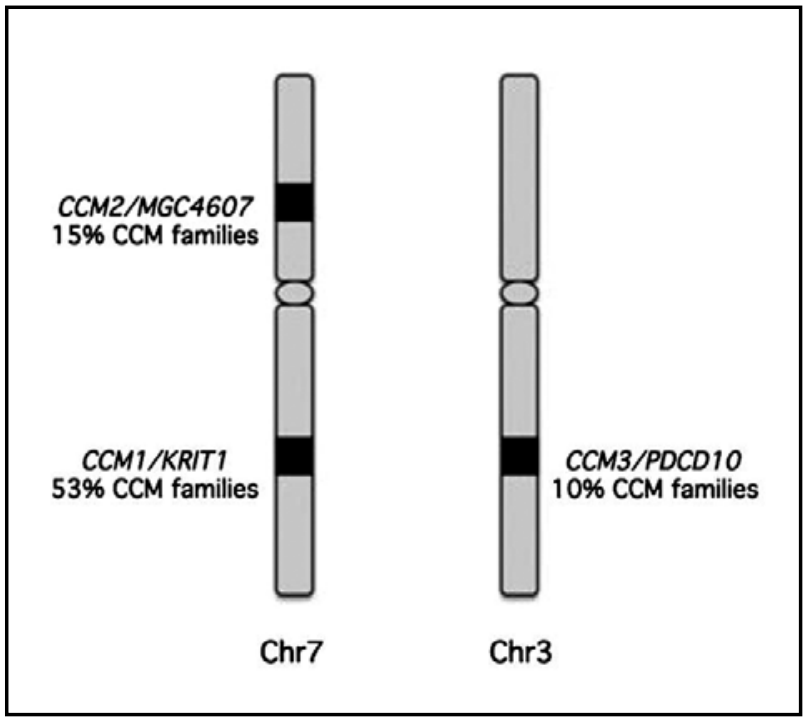

Figura 1. Loci CCM y sus genes, que codifican las proteínas KRIT1, MGC4607 (malcavernina) y PDCD10.

oscura, que semeja un berry (morfología en frambuesa o palomita de maíz), rodeado por una cápsula de cerebro gliótico.

En la microscopía, la MC tiene células endoteliales con uniones estrechas pobremente formadas, con frecuentes hendiduras entre las células. Los pericitos, precursores de células de músculo liso, están en insuficiente cantidad. Además no se encuentran podocitos de astrocitos ni tejido nervioso normal dentro de la lesión ${ }^{(17,18)}$.

Desde mediados de la década de los ' 90 , tras el primer reporte de Ciricillo, ha aparecido evidencia que involucra a la radioterapia en el desarrollo de MC, principalmente la utilizada en el manejo de neoplasias cerebrales infantiles ${ }^{(19)}$. Las neoplasias más frecuentemente ligadas a este proceso son los gliomas, meduloblastomas y leucemia linfoblástica aguda, sin poder determinarse con certeza el proceso patológico por el cual se desarrollan, ni en humanos ni en modelos animales y las dosis responsables oscilan alrededor de los 30 Gy. Los periodos de aparición de tales neoplasias son tan breves como 12 meses o tan extensos como 26 años. La ubicación es claramente intracraneal, claramente intracraneal, en relación al campo de irradiación. Los de ubicación medular reportados no superan la decena; de éstos, la gran mayoría son identificados en el segmento cervical.

En la actualidad, continúan generándose publicaciones que asocian la aparición de angiomas cavernosos de novo en pacientes sometidos a radiocirugía (quienes poseían una RM previa sin evidencias de una MC), con dosis cercanas a los 5 Gy. Pese a que ello se supone como cofactor en su aparición, ya hay datos sobre $\mathrm{MC}$ que han debido tratarse, paradójicamente, con radiocirugía ${ }^{(20-23)}$. 


\section{Manifestaciones clínicas}

Suelen manifestarse con mayor frecuencia en el grupo etario que va entre los 40 y 60 años. Su curso clínico es variable debido a la naturaleza dinámica de la MC.

Cohortes seguidas prospectivamente demostraron que el mayor porcentaje de los sujetos es asintomático, siendo estas lesiones un hallazgo incidental. Dentro de aquella minoría sintomática, los hallazgos son heterogéneos, dependiendo de su localización y de si se asocian o no a hemorragia, encontrándose manifestaciones como crisis convulsivas (40-50\%), focalización neurológica, cefaleas (30\%) y hemorragia intracraneal $(10-25 \%)^{(24,25)}$.

Las microhemorragias que presentan habitualmente son subclínicas, con una frecuencia de $0.5-1 \%$ por año, siendo más cercano al valor inferior en los casos esporádicos y tendiendo al $1 \%$ en los de tipo familiar.

Un factor de riesgo para una hemorragia es la existencia de una hemorragia previa. El riesgo de presentar una nueva hemorragia en pacientes con hemorragia sintomática se estima en un $25 \%$ dentro de un año(4, 26).

Mención especial requieren las MC ubicadas en tronco cerebral, pues la aparición de una segunda hemorragia a menudo tiene un efecto catastrófico. La probabilidad de una segunda hemorragia, variable según las publicaciones, se estima en $75 \%$ dentro de un período de 26 meses y con riesgo de un nueva hemorragia en $21 \%$ por año ${ }^{(27,28)}$.

Kondziolka, en un análisis por subgrupos describe una tasa aumentada de hemorragia de $4.5 \%$ por año en pacientes sintomáticos, la que es superior en comparación al $0.7 \%$ global $^{(29)}$.

Además, está demostrado que un tamaño superior a $10 \mathrm{~mm}$ se correlaciona con una mayor posibilidad de sangrado (OR 3.48; IC 95\% 1.3-9.4)(30).

Por otra parte, la recuperación posterior a un evento sintomático es pobre y no está directamente relacionada a la presencia o ausencia de hemorragia en las imágenes. Sólo un 37\% presenta recuperación completa de los déficits focales, $36 \%$ tienen recuperación parcial y $27 \%$ no presentan ninguna mejoría ${ }^{(31)}$.

\section{Hallazgos imagenológicos}

En los tiempos previos al desarrollo de TC y RM se le conocía como una "malformación vascular oculta", dado que las angiografías cerebrales no eran capaces de demostrar su existencia por el muy bajo flujo intralesional que poseen. De tal modo que en no más del $10 \%$ de los estudios se lograba diagnosticar esta malformación, siendo visible en las fases capilar o venosa como un débil realce ${ }^{(32)}$ (Figura 2).

Los avances en la tecnología permitieron intentar su detección en TC. Sin embargo, este método tiene una tasa de falsos negativos entre $30-40 \%$, ya que habitualmente miden menos de $3 \mathrm{~cm}$, tienen una atenuación similar al parénquima circundante y experimentan escaso o nulo realce tras el uso de contraste endovenoso. Su rendimiento mejora cuando se presentan levemente hiperdensos y asociados a calcificaciones $^{(6)}$ (Figura 3 ).

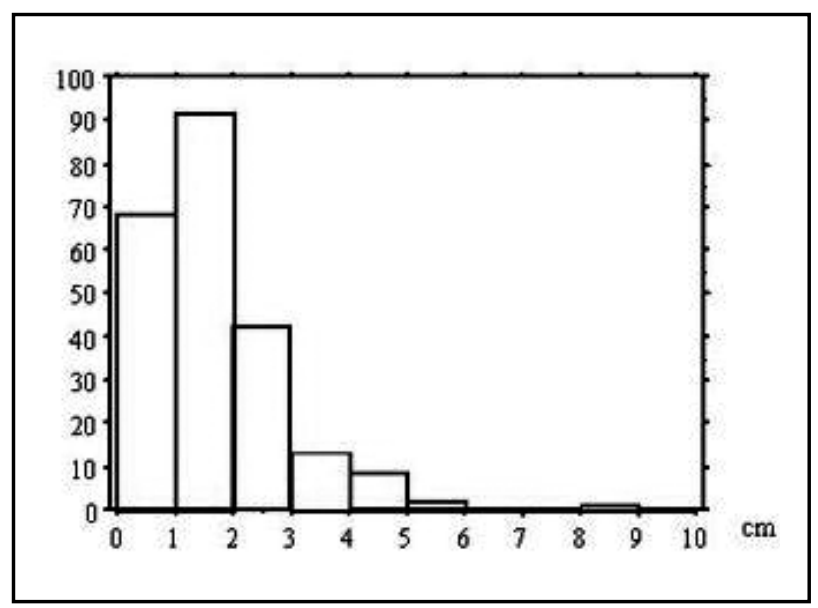

Figura 2. Tamaño de las MC en 728 casos en 347 pacientes.

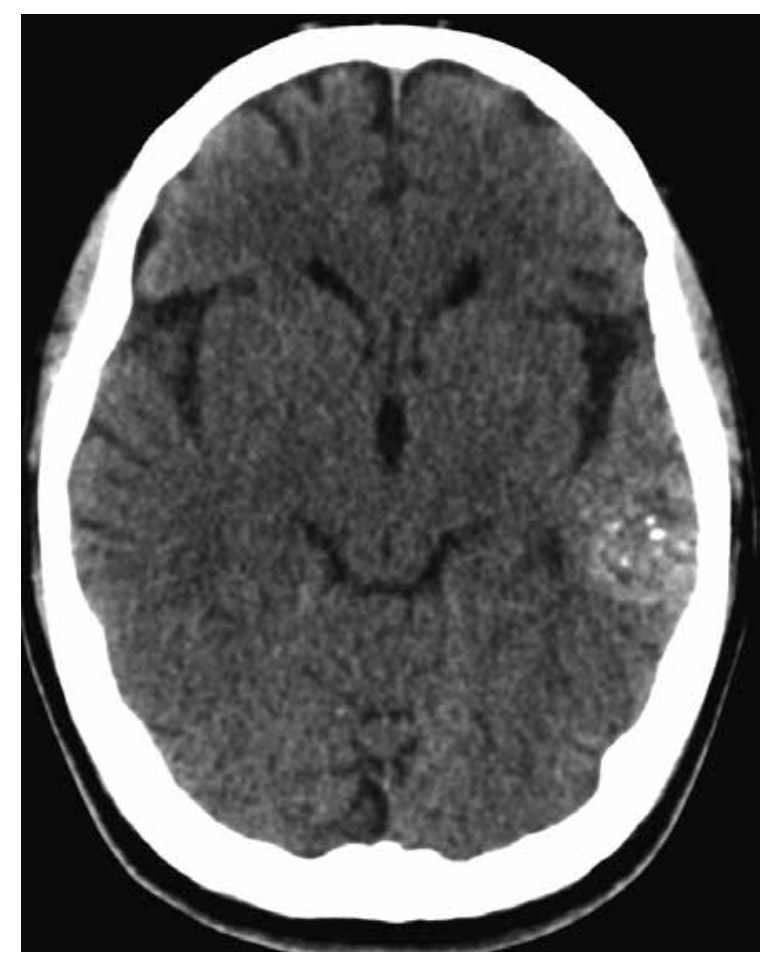

Figura 3. Imagen clásica de la MC cortical densa con calcificaciones en TC, en lóbulo temporal izquierdo.

Posteriormente, la RM se convirtió paulatinamente en la técnica de elección en la pesquisa de estas lesiones, las que se muestran como imágenes bien delimitadas, lobuladas, con morfología de "palomita de maíz", dada por un área central con distintas intensidades de señal que dan cuenta de los restos 
hemáticos en diversos estadios de degradación, incluyendo niveles líquido-líquido de componentes hemáticos en el interior de las loculaciones. Cuando superan los $3 \mathrm{~cm}$, es frecuente encontrar focos hipointensos puntiformes (black dots) en imágenes T2 GRE. La lesión típicamente está marginada por un anillo de hemosiderina, hipointenso en secuencias $\mathrm{T} 2$, y especialmente identificable en secuencias $\mathrm{T} 2^{*}$ o GRE y, de aparición posterior, en la secuencia ponderada en susceptibilidad (SWI) ${ }^{(33)}$ (Figura 4).

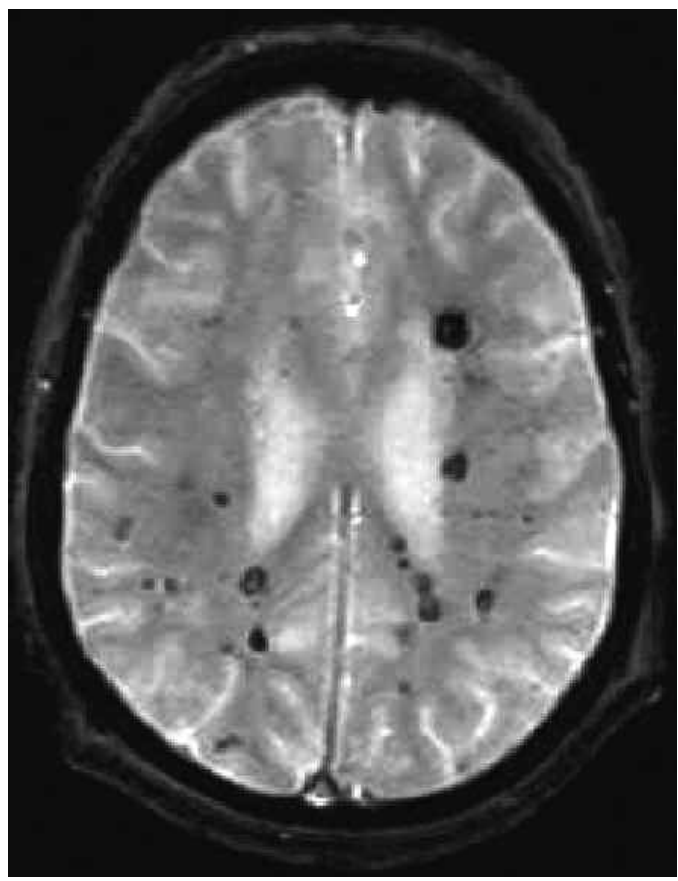

Figura 4. Múltiples focos de $M C$ en $R M$, hipointensos en secuencia $T 2{ }^{*}$ o GRE.

En estas dos últimas secuencias, es posible hacer más notorias las MC a través del efecto "blooming", originado por los artefactos de susceptibilidad magnética determinados por los productos de degradación hemáticos, que en secuencias convencionales de RM pudiesen haber pasado desapercibidas. De ahí, que sean las secuencias recomendadas para detección y seguimiento, sobretodo en los casos del tipo familiar (Figuras 5, 6, 7 y 8).

Zabramski ha propuesto clasificar estas lesiones en distintas categorías en base a características en $\mathrm{RM}^{(34)}$ (Tabla I).

En el diagnóstico diferencial de las malformaciones cavernosas, se deben tener presente:

- Malformaciones arteriovenosas, que pueden producir edema o efecto de masa, pero no tienen las diversas fases de degradación hemoglobínica.

- Tumores hemorrágicos con anillo de hemosiderina incompleto y distintos estados de degradación de la hemoglobina, que presentan realce intenso del componente tumoral.
- Tumores calcificados como el oligodendroglioma, que habitualmente muestran realce irregular, parchado.

- Granulomas calcificados.

- Focos hemosiderínicos de otra etiología (por ejemplo hipertensión arterial o angiopatía amiloidea).

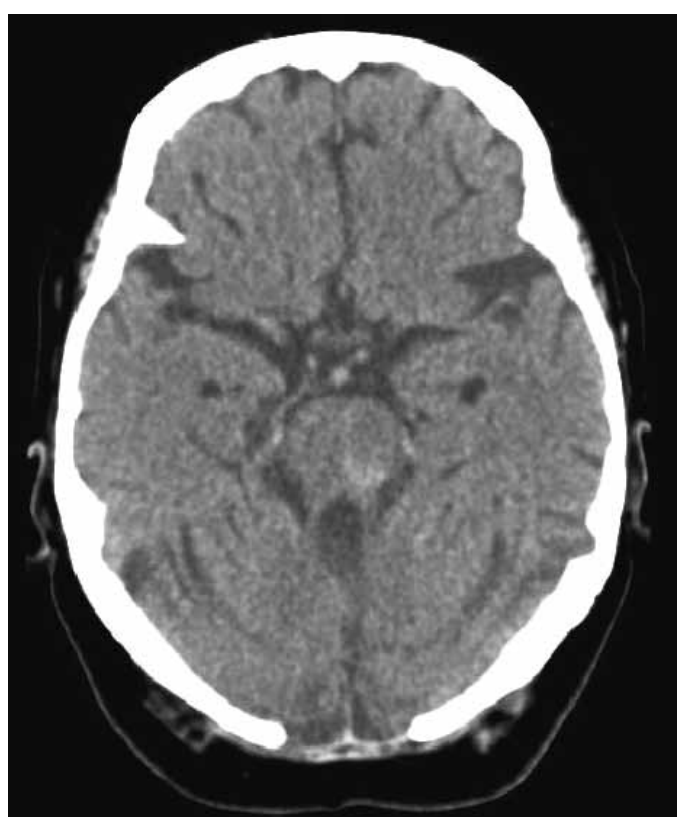

Figura 5. MC en tronco cerebral. Corte axial de TC.

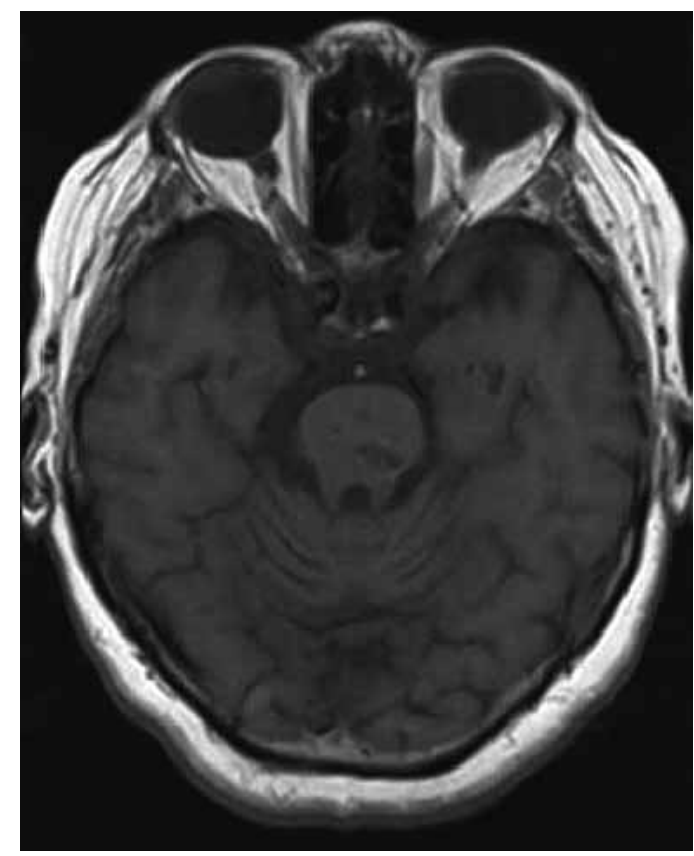

Figura 6. MC en tronco cerebral. Secuencia T1. 


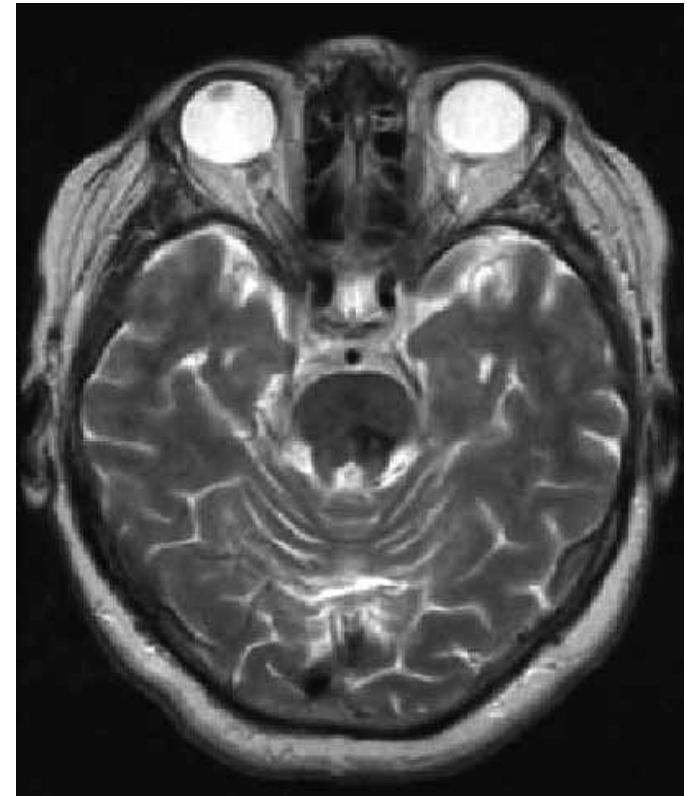

Figura 7. MC en tronco cerebral. Secuencia T2 TSE.

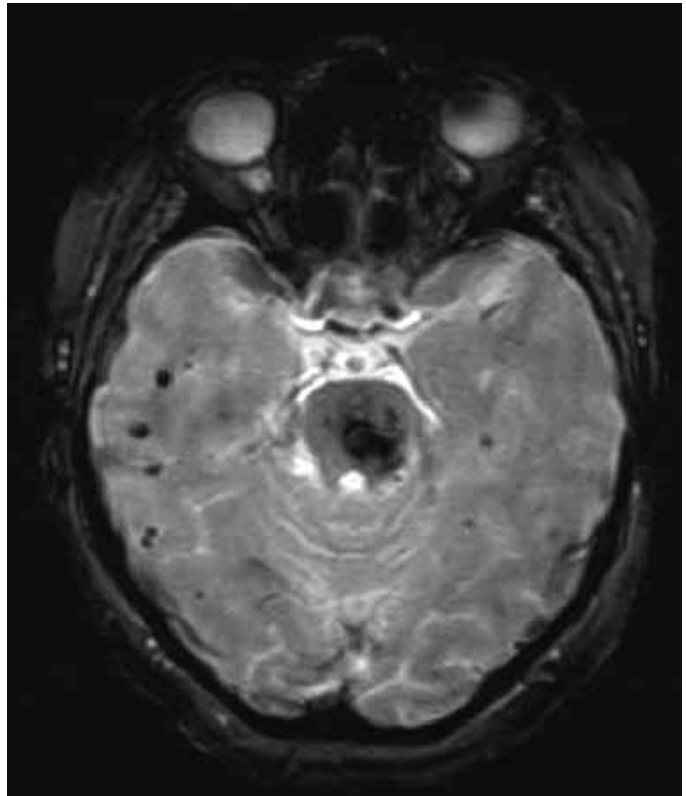

Figura 8. MC en tronco cerebral. Secuencia T2*..

Tabla I. Clasificación por RM de las malformaciones cavernomatosas.

\begin{tabular}{|c|c|c|}
\hline Lesión & Señal en RM & Característica Patológica \\
\hline Tipo I & $\begin{array}{l}\text { T1: Núcleo hiperintenso } \\
\text { T2: Núcleo hiper/hipointenso con anillo } \\
\text { hipointenso }\end{array}$ & $\begin{array}{l}\text { Hemorragia subaguda, rodeada por un anillo } \\
\text { de hemosiderina, macrófagos y cerebro gliótico. }\end{array}$ \\
\hline Tipo II & $\begin{array}{l}\text { T1: Núcleo con señal mixta reticulada } \\
\text { T2: Núcleo con señal mixta reticulada } \\
\text { con anillo hipointenso }\end{array}$ & $\begin{array}{l}\text { Áreas loculadas de hemorragia y trombosis } \\
\text { de distinta temporalidad, rodeada por cerebro } \\
\text { con gliosis y hemosiderina; en las lesiones } \\
\text { grandes se puede ver zonas de calcificación. }\end{array}$ \\
\hline Tipo III & $\begin{array}{l}\text { T1: Iso/hipointenso } \\
\text { T2: Hipointensidad con un anillo hipointenso } \\
\text { que magnifica el tamaño de la lesión } \\
\text { T2*: Hipointensidad magnificada respecto a T2 } \\
\text { (blooming) }\end{array}$ & $\begin{array}{l}\text { Hemorragia en fase crónica con hemosiderina } \\
\text { dentro y alrededor de la lesión. }\end{array}$ \\
\hline Tipo IV & $\begin{array}{l}\text { T1: Lesión poco o no visible } \\
\text { T2: Lesión poco o no visible } \\
\text { T2*: Lesiones hipointensas puntiformes }\end{array}$ & $\begin{array}{l}\text { Las lesiones de esta categoría corresponden } \\
\text { a telangectasias. }\end{array}$ \\
\hline
\end{tabular}

\section{MC medulares}

Se describen como lesiones raras, con reportes de casos que apenas superan el centenar de pacientes en casi un siglo. Inicialmente contabilizados en base a $\mathrm{MC}$ resueltas quirúrgicamente, posteriormente se suman las demostradas por imágenes tras la aparición de la RM, lo que permitió la detección de casos en fase preclínica.

Dan cuenta del 5-12\% de las anormalidades vas- culares espinales, con una distribución más frecuente en el segmento cervical, seguido por los segmentos dorsal y lumbar. Habitualmente diagnosticadas en la quinta década de la vida y sin predominio de género, con una tasa de sangrado estimado en $1.4 \%$ por lesión/año (0.4-2\% por año). Se presume que las eventuales hemorragias ocurren a baja presión y por ello las manifestaciones clínicas no son tan dramáticas como las provenientes de lesiones arteriales ${ }^{(25,35,36)}$. 
La historia natural de las MC medulares es confusa. La mayoría de las series quirúrgicas han reportado altas tasas de sangrado en pacientes con manejo quirúrgico, mientras que hay informes contrapuestos con series que muestran una importante menor frecuencia de hemorragia en pacientes manejados de forma conservadora ${ }^{(37)}$.

\section{Localizaciones atípicas}

La MC con poca frecuencia se encuentra en localización extracerebral. En este caso, tendrán una presentación clínica, hallazgos imagenológicos

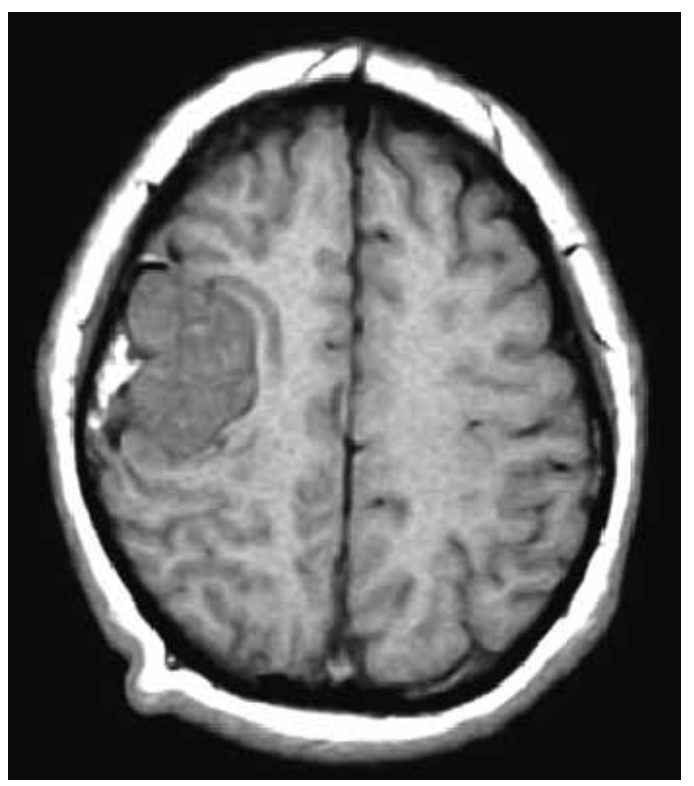

Figura 9. MC en localización atípica extraaxial intradural. Secuencia ponderada en T1.

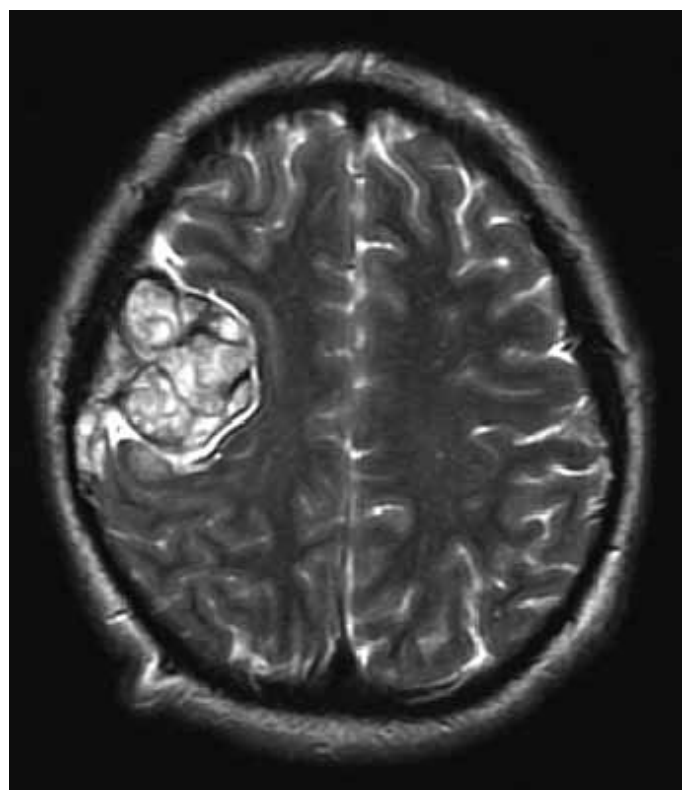

Figura 10. MC en localización atípica extraaxial intradural. Secuencia ponderada en T2. y resultados post tratamiento diferentes (Figuras 9 , 10, 11 y 12).

La MC del seno cavernoso corresponde a menos del $1 \%$ de las masas paraselares, tiene predilección por el sexo femenino (7:1) y se manifiesta alrededor de la cuarta década de vida. Su origen puede estar dentro del seno cavernoso o en las estructuras que lo rodean, creciendo lenta y progresivamente. Los síntomas clínicos más habituales en esta localización son cefalea, dolor retroorbitario, parálisis de nervios craneanos con oftalmoplejia, proptosis, anisocoria y cambios sensoriales faciales, pudiendo haber exacer-

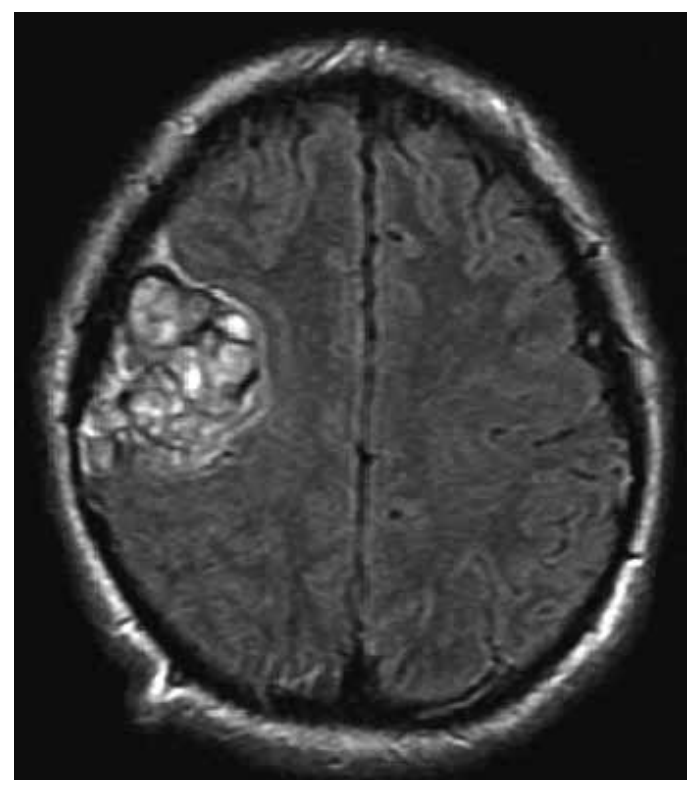

Figura 11. MC en localización atípica extraaxial intradural. Secuencia FLAIR.

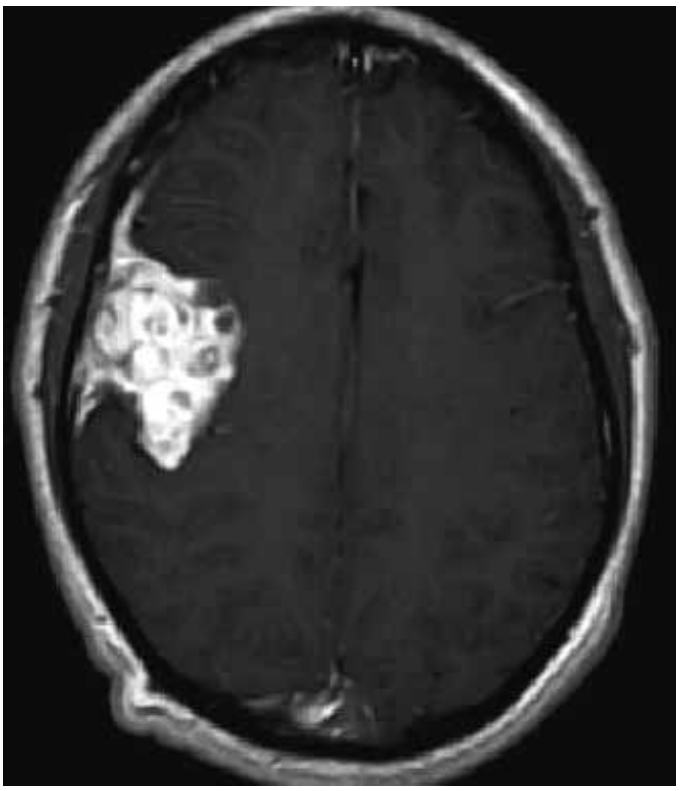

Figura 12. MC en localización atípica extraaxial intradural. Secuencia T1 con gadolinio. 
bación durante el embarazo. Pueden evolucionar con trombosis, degeneración hialina y calcificaciones ${ }^{(38)}$.

El ángulo pontocerebeloso es otra localización inhabitual de las MC. En esta ubicación pueden presentarse como masas sólidas que se extienden hacia el conducto auditivo interno o, menos comúnmente, como lesiones quísticas. En la degeneración quística estarían involucrados mecanismos tales como hemorragias menores recurrentes sinusoidales o de neocapilares, las que aumentarían la presión osmótica local, arrastrando fluido gradualmente dentro de la MC, favoreciendo dicha degeneración quística y su progresivo crecimiento ${ }^{(39)}$.

\section{Manejo}

No obstante el foco de la revisión está puesto en la historia natural y su correlato imagenológico, podemos mencionar que el abordaje terapéutico depende de la presencia de convulsiones, localización y de la apariencia radiológica de las hemorragias. Generalmente el manejo es conservador, interviniéndose en casos seleccionados, ya sea con cirugía tradicional o radiocirugía (Gamma knife). En los casos en que estas opciones no sean posibles, como en lesiones de tronco con sangrado recurrente, se ha descrito el manejo con factor VI la recombinante endovenoso, con lo que se obtiene buenos resultados ${ }^{(25,40)}$.

\section{Conclusión}

La MC es una de las malformaciones vasculares comunes del SNC y que, pese a no ser habitualmente sintomática, es causa de convulsiones y déficit focales. Si se sospecha o se detecta por otro método, es recomendable su plena identificación y caracterización con RM, también utilizable en los casos particulares que ameriten seguimiento.

Pese a que las ubicaciones clásicas son altamente frecuentes, no se debe perder de vista que éstas pueden originarse en cualquier otro lugar, razón por la cual en ubicaciones inhabituales es el patrón imagenológico típico lo que contribuirá a dilucidar el diagnóstico diferencial.

Es importante estar atentos a los avances que las ciencias básicas puedan lograr en el estudio y comprensión molecular de las MC, ya que pudieran cambiar la historia natural de la enfermedad y de su tratamiento.

\section{Bibliografía}

1. Bigner D, McLendon R, Bruner J. Russell \& Rubinstein's Pathology of Tumors of the Nervous System. 6th ed. London: Arnold, 1998.

2. Batra S, Lin D, Recinos P, Zhang J, Rigamonti D. Cavernous malformations: natural history, diagnosis and treatment. Nat. Rev. Neurol 2009; 5, 659-670.

3. Otten P, Pizzolato G, Rilliet B,Berney J. 131 cases of cavernous angioma of the CNS, discovered by retros- pective analysis of 24,535 autopsies. Neurochirurgie 1989; 35: 128-131.

4. Robinson J, Awad I, Little J. Natural history of the cavernous angioma. J Neurosurg 1991; 75: 709-714.

5. Brunon J, Nuti C. Natural history of cavernomas of the central nervous system. Neurochirurgie 2007; 53 : 122-130.

6. El-Koussya M, Stepperb F,Sprenga A, Lukesc A, GraIlaa J,Brekenfelda C, Sturzeneggerb M, Schrotha G. Incidence, clinical presentation and imaging findings of cavernous malformations of the CNS. SwissMedWkly. 2011; 141: w13172.

7. Rigamonti D, Hadley M, Drayer B. Cerebral cavernous malformations: incidence and familial occurrence. $\mathrm{N}$ Engl J Med 1988; 319: 343-347.

8. Günel M, Awad I, Finberg K, Anson J,Lifton R. A founder mutation as a cause of cerebral cavernous malformation in Hispanic Americans. N Engl J Med 1996; 334: 946-951.

9. Liu J, Lu Y, Raslan A,Gultekin H, DeLashaw J.B. Cavernous malformations of the optic pathway and hypothalamus: analysis of 65 cases in the literature. NeurosurgFocus 2010; 29: e17-e25.

10. Albanese A,Sturiale C,D'Alessandris Q, Capone G, Maira G. Calcified extra-axial cavernoma involving lower cranial nerves: technical case report. Neurosurgery. 2009; 64: 135-136.

11. Ito $M$, Kamiyama $H$, Nakamura $T$, Nakajima $H$, Tokugawa J.Dural cavernous hemangioma of the cerebellar falx. Neurol Med Chir (Tokyo) 2009; 49: 410-412.

12. Kivelev J,Niemelä M, Kivisaari R,Hernesniemi J. Intraventricular cerebral cavernomas: a series of 12 patients and review of the literatura. J Neurosurg 2010; 112: 140-149.

13. Omodaka S,Fujimura M, Endo T, Inoue T, Shimizu H, Tominaga T. De novo formation of orbital cavernous malformation 9 years after surgical management of dural arteriovenous fistula in the anterior middle fossa. Case Report. Neurol Med Chir (Tokyo) 2010; 50: 324327.

14. Krisht K, Whitehead K,Niazi T,Couldwell W. The pathogenetic features of cerebral cavernous malformations: a comprehensive review with therapeutic implications. Neurosurg Focus. 2010; 29: E2-E7.

15. Riant F,Bergametti F,Ayrignac X,Boulday G,TournierLasserve. E. Recent insights into cerebral cavernous malformations: the molecular genetics of CCM. FEBS Journal 2010; 277: 1070-1075.

16. Whitehead K, Chan A,Navankasattusas S,Koh W, London N, Ling J. The cerebral cavernous malformation signaling pathway promotes vascular integrity via Rho GTPases. NatMed 2009; 15: 177-184.

17. Zawistowski J,Stalheim L,Uhlik M,Abell A,Ancrile B, Johnson G. CCM1 and CCM2 protein interactions in cell signaling: implications for cerebral cavernous malformations pathogenesis. Hum Mol Genet 2005; 14: 2521-2531.

18. Stockton R,Shenkar R,Awad I, Ginsberg M. Cerebral cavernous malformations proteins inhibit Rho kinase to stabilize vascular integrity. J ExpMed 2010; 207: 881-896.

19. Ciricillo S, Cogen P, Edwards M. Pediatric cryptic vascular malformations: presentation, diagnosis and treatment. PediatrNeurosurg 1994; 20: 137-147. 
20. Nimjee S, Powers C,Bulsara K, Review of the literature on de novo formation of cavernous malformations of the central nervous system after radiation therapy, Neurosurg Focus. 2006; 21: e4-e10.

21. Strenger V, Sovinz P, Lackner H, Dornbusch H, Lingitz $\mathrm{H}$, Eder $\mathrm{H}$, et al. Intracerebral Cavernous Hemangioma after Cranial Irradiation in Childhood. StrahlentherOnkol 2008; 184: 276-280.

22. Heckl S, Aschoff A, Kunze S. Radiation-induced cavernous hemangiomas of the brain: A late effect predominantly in children. Cancer 2002; 94: 32853291.

23. Park Y, Chang J, Kim S, Chang J, Park Y. Radiosurgery for Radiosurgery-induced Cavernous Malformation. WorldNeurosurg 2011; 75: 94-98.

24. Josephson C, Leach J, Duncan R, Roberts R,Counsell C, Al-Shahi Salman R. Seizure risk from cavernous or arteriovenous malformations: Prospective populationbased study. Neurology 2011; 76: 1548-1554.

25. Ramírez-Zamora A, Biller J. Brainstem Cavernous Malformatios: A Review with two cases reports. Arq Neuropsiquiatr 2009; 67: 917-921.

26. Pozzati E, Acciarri N, Tognetti F. Growth, subsequent bleeding and de novo appearance of cerebral cavernous malformations. Neurosurgery 1996; 38: 662-670.

27. Sakai N, Yamada H, Tanigawara T. Surgical treatment of cavernous angioma involving the braistem and review of literature. Acta Neurochir 1991; 113: 138-143.

28. Fritschi J, Reulen H, Spetzler R. Cavernous malformation of the brain stem. A review of 139 cases. Acta Neurochir 1994; 130: 35-46.

29. Kondziolka D, Lunsford L,Kestle J. The natural history of cerebral cavernous malformations. J Neurosurg 1995; 83: 820-824.

30. Kupersmith M, Kalish H, Epstein F, Yu G, Berenstein A, Woo H, et al. Natural History of Brainstem Cavernous Malformations. Neurosurgery 2001; 48: 47-54.
31. Porter P,Wilinsky R, Harper W, Wallace W. Cerebral cavernous malformations: Natural history and prognosis after clinical deterioration with or without hemorrhage. J Neurosurg 1997; 87: 190-197.

32. Tagle P, Huete I, Mendez J, Del Villar S. Intracranial cavernous angioma: presentation and management. J Neurosurg 1986; 64: 720-723.

33. De Champfleur N, Langlois C, Ankenbrandt W, Le Bars E, Leroy M, Duffau H, Bonafé A, Jaffe J, Awad A, Labauge P.Neurosurgery 2011; 68: 641-647.

34. Zabramski J, Wascher T, Spetzler R. The natural history of familial cavernous malformations: results of an ongoing study. J Neurosurg 1994; 80: 422-432.

35. Cosgrove G, Bertrand G, Fontaine F, Robitaille Y, Melanson D. Cavernous angiomas of the spinal cord. J Neurosurg 1998; 68: 31-36.

36. Zevgaridis D, Medele R, Hamburger C, Steiger $H$, Reulen R. Cavernous Haemangiomas of the Spinal Cord. A Review of 117 Cases. ActaNeurochir (Wien) 1999; 141: 237-245.

37. Kharkar S, Shuck J, Conway J,Rigamonti D. The natural history of conservatively managed symptomatic intramedullary spinal cord cavernomas. Neurosurgery 2007; 60, 865-872.

38. Salanitri G, Stuckey S, Murphy M. Extracerebral Cavernous Hemangioma of the Cavernoues Sinus: Diagnosis with MR Imaging and Labeled Red Cell Blood Pool Scintigraphy. Am J Neuroradiol 2004; 25: 280-284.

39. Huang H, Xu K, Qu L, Li Y, Yu J. Cystic cavernous malformation of the cerebellopontine angle: Case report and literature review. World J SurgOncol 2011; 9: 36.

40. Engelhardt K, Brenneis C, Pfausler B, Beer R, Brössner G, Helbok R, et al. rFVIla - for acute rebleeding of a cerebral cavernous malformation. Eur J Neurol 2007, 14: 117-120. 